\title{
Periapical Wires Result in Less Curve Correction Than Pedicle Screw Constructs in Idiopathic Scoliosis
}

\author{
Valentine R. Esposito ${ }^{1}$, Brian L. Dial ${ }^{2}$, Robert D. Fitch ${ }^{2}$, Robert K. Lark ${ }^{2}$ \\ ${ }^{1}$ Duke University School of Medicine, Durham, NC, USA \\ ${ }^{2}$ Department of Orthopedic Surgery, Duke University Medical Center, Durham, NC, USA
}

\section{Study Design: Retrospective study.}

Purpose: The objective of this study was to compare percent correction between apical and periapical pedicle screw (PS) and sublaminar wire constructs for Cobb correction and coronal balance correction.

Overview of Literature: The current gold standard for deformity correction in adolescent idiopathic scoliosis (AIS) are PS constructs. Sublaminar wires provide an alternative means of fixation when PS fixation cannot be safely performed. Two previous studies have compared percent curve correction between sublaminar wires and PSs, with conflicting conclusions.

Methods: The study was a retrospective review of Lenke type 1 curves with minimum follow-up of at least 1 year. Cases were divided into two groups: constructs using apical/periapical sublaminar wires (SL group) versus PS only constructs (PS group). Percent Cobb correction and coronal balance were compared between the two groups at 1 year. A multivariable regression model was used to determine the impact of apical/periapical wires on percent Cobb correction and coronal balance at 1 year when accounting for additional variables.

Results: The cohort included 71 patients who were predominantly female (80.2\%), with average age of 14.2 years. Only 21 (29.5\%) of constructs utilized apical/periapical sublaminar wires. There was a significant difference in percent Cobb correction at 1 year for the PS and SL groups $(70.26 \%$ vs. $60.09 \%, p=0.05)$. No difference was observed in overall coronal balance. A multivariable model revealed that apical/periapical wires were negatively associated with percent Cobb correction at 1 year (coefficient=-8.49, $p=0.023$ ), while total implant density of the construct was positively associated with correction (coefficient=24.2, $p<0.001$ ).

Conclusions: Use of PSs in the apical and periapical zones resulted in improved percent Cobb correction at 1 year in patients with AIS Lenke type 1 curves. Sublaminar wires remain a useful surgical option and result in equivalent coronal balance compared to PSs.

Keywords: Scoliosis; Sublaminar wires; Curve correction

\section{Introduction}

Adolescent idiopathic scoliosis (AIS) is a three-dimensional spinal deformity of coronal translation, sagittal hypokyphosis, and axial rotation. Modern surgery for AIS originated in the 1950s with the use of the Harrington rod. The evolution of spinal fixation included the use of sublaminar wires, Cotrel-Dubousset segmental

Received Jan 2, 2019; Revised Feb 9, 2019; Accepted Mar 19, 2019

Corresponding author: Robert K. Lark

Department of Orthopaedics and Pediatrics, Duke University Medical Center, 3000 Erwin Road, DUMC 2809, Durham, NC 27710, USA

Tel: +1-919-681-7605, Fax:+1-919-681-7618, E-mail: robert.lark@duke.edu 
instrumentation, and segmental pedicle screw (PS) instrumentation. Fixation with PSs compared to hybrid or all-hook constructs results in improved coronal and axial deformity correction, minimizes the required number of levels fused, and limits the need for anterior surgery [1-8]. In addition to improving the ability to correct deformity, PSs have been shown to be safe. A prospective cohort study of 1,301 children undergoing spinal fusion and instrumentation for AIS yielded a neurologic complication rate of $0.69 \%$. Of the nine neural complications, only two resulted from medial placement of PSs [9].

Certain situations require alternative means of fixation despite the effectiveness and overall safety of PSs. Specifically, alternative implants should be considered when PS placement is unsafe due to the patient's pedicle morphology, in the setting of pedicle fracture, or for salvage of a previously misplaced PS. In AIS, thoracic pedicles at the apex of the deformity have an increased rate of dysmorphism. Additionally, there is asymmetry between the concave and convex pedicles, with concave pedicles having narrower diameters in both the transverse and sagittal planes $[2,10]$. At the apex of the curve, the position of the spinal cord is displaced, and it is located along the medial border of the concave pedicles. Medial wall breach of an apical concave pedicle is at increased risk for spinal cord injury [11]. The location of the aorta also needs to be considered, as damage to the aorta during PS placement would result in catastrophic vascular injury. In AIS, the thoracic aorta is displaced posterolaterally, and lateral wall breach of left sided pedicles could result in aortic injury [12]. In certain situations, the morphology of the pedicle and surrounding anatomy, specifically concave pedicles near the apex of the deformity, may not allow for safe PS placement, and alternative means of fixation can be considered.

Given the potential risks associated with PS placement in pedicles with morphology that does not safely accommodate a PS, it is critical to evaluate the comparative efficacy of available alternative implants. Currently, other than PSs, the most commonly utilized implants include transverse process hooks, pedicle hooks, laminar hooks, sublaminar bands, and sublaminar wires. All PS constructs allow for greater deformity correction compared to hybrid constructs. Additionally, there is limited evidence in the literature addressing how the utilization of alternative implants solely in the apical pedicles impacts curve correction.
The purpose of this study was to determine the comparative efficacy of sublaminar wires relative to PSs in the apical and periapical zones for patients with AIS Lenke type 1 curves. We hypothesized that use of sublaminar wires in the apical and periapical zones would demonstrate comparable curve correction when compared with PS constructs.

\section{Materials and Methods}

\section{Patients}

Following Institutional Review Board approval we queried a Duke University Medical Center database for pediatric patients who underwent posterior spinal fusion (PSF) for AIS between 2005 and 2015, all treated by a single surgeon at our institution. Our system was able to screen all patients seen at our institution after 1996, with the ability to set the inclusion and exclusion criteria prior to running a structured search to produce a cohort of patients [13]. Patients who were diagnosed with neuromuscular scoliosis, congenital scoliosis, juvenile scoliosis, did not have a minimum of 1 year of follow-up, and with AIS curves other than Lenke type 1 were excluded from our study. To obtain further demographic, operative, and clinical information, cases meeting the inclusion criteria were subjected to chart review. We retrospectively reviewed 273 patients with PSF for scoliosis identified by our database. Following radiographic and chart review, 94 patients were identified as having Lenke type 1 curves. The final cohort consisted of 71 patients, as 23 patients had insufficient imaging or follow-up for inclusion.

Patients were divided into two groups. The SL group included constructs utilizing apical and periapical sublaminar wires, and the PS group included constructs that used all PSs within the apical and periapical zones (Figs. 1, 2). The primary surgeon's decision to utilize sublaminar wires was made intra-operatively based on difficult PS placement due to dysplastic pedicles, pedicle fracture, or pedicle wall breach. The apical zone was defined as the apical vertebra $+/-1$ vertebra (Fig. 3). The periapical zones were defined as the vertebral bodies between the apical zones and the cephalad or caudad zones (Fig. 3). The following variables were compared between the two groups: Lenke curve type, curve flexibility, pre- and postoperative Cobb angles, percent Cobb correction, pre- and postoperative coronal balance, implant density, and estimated blood loss 

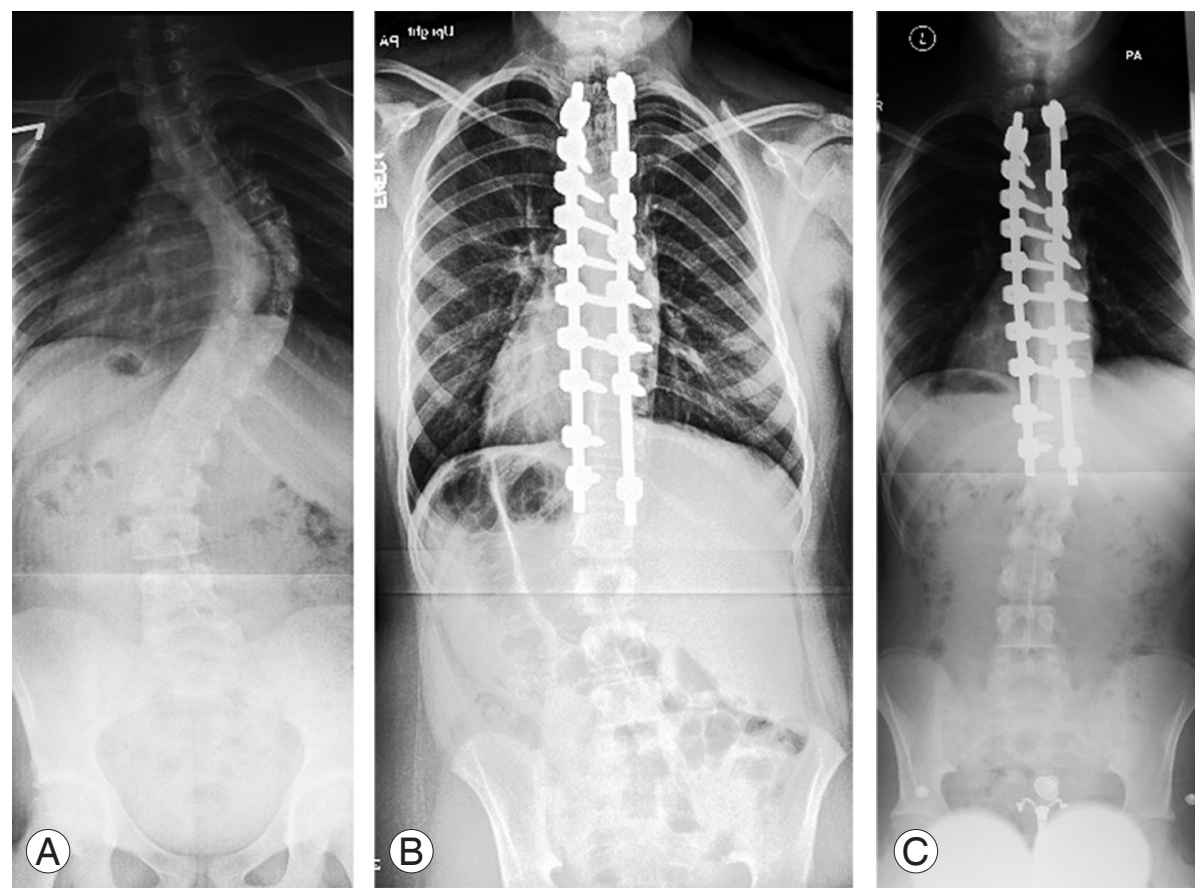

Fig. 1. Pre- and postoperative radiographs of patient in pedicle screw group. (A) Preoperative radiograph, (B) postoperative radiograph, and (C) 1-year follow-up radiograph.
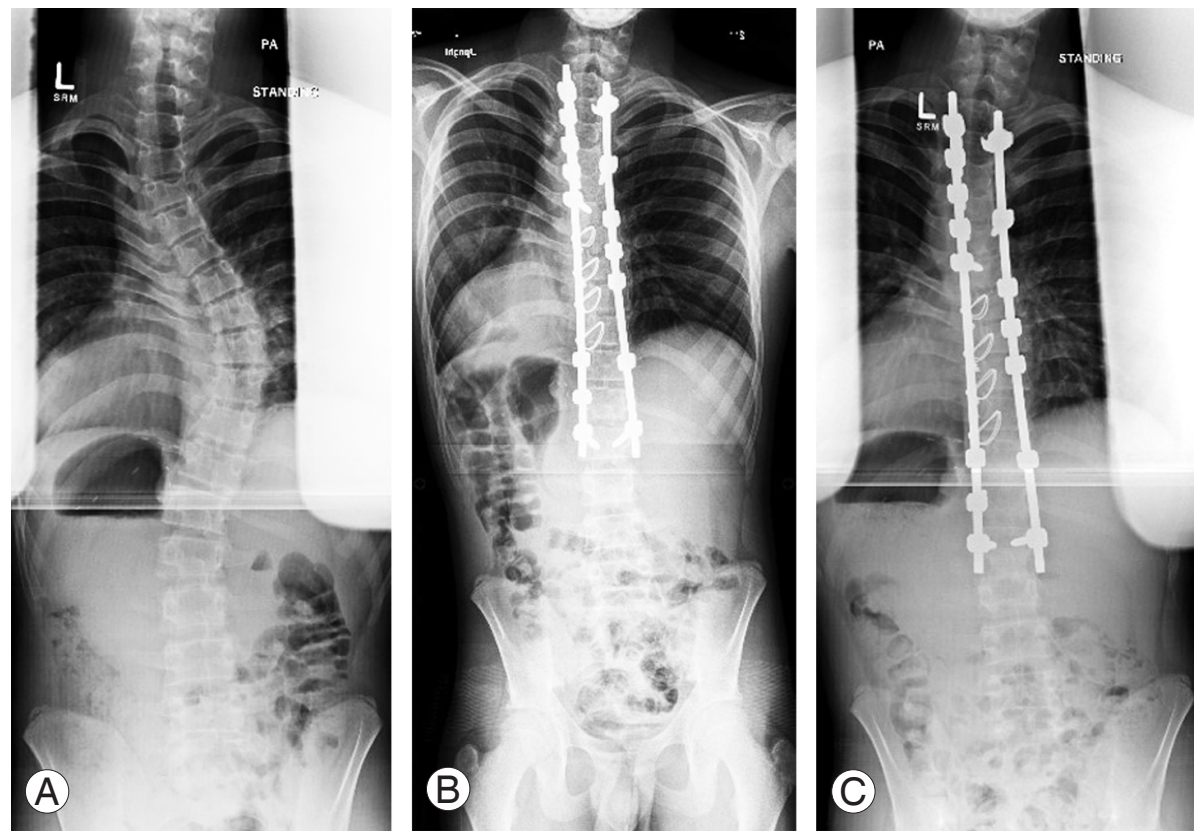

Fig. 2. Pre- and postoperative radiographs of patient in the sublaminar wire group. (A) Preoperative radiograph, (B) postoperative radiograph, and (C) 1-year follow-up radiograph.

(EBL). Implant density was defined as the average number of fixation points per level fused. These variables were first compared between the two groups utilizing univariate analysis. Coronal balance was defined as the absolute value of the distance between the $\mathrm{C} 7$ plumb line and the central sacral vertical line (CSVL).

A multivariable regression model was used to examine the impact of apical/periapical sublaminar wires, curve flexibility, number of levels fused, and total implant density on percent Cobb correction and coronal balance at 1 year. 


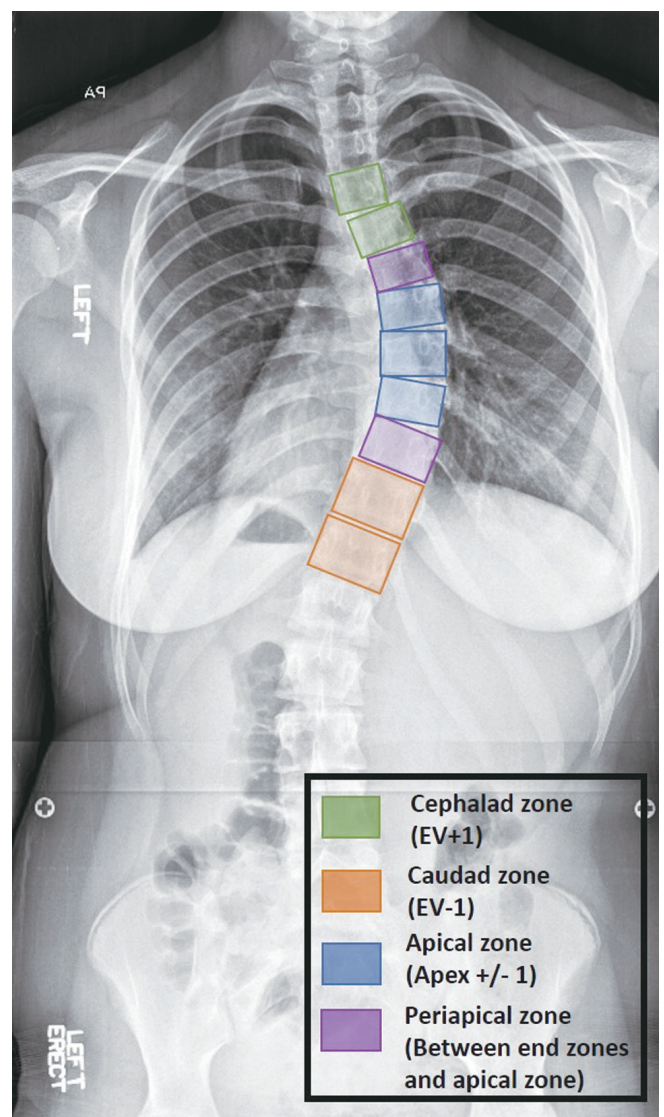

Fig. 3. Representation of the zones along the spinal fusion. Apical zone is the apex $+/-1$ level, cephalad zone is the upper instrumented vertebra and one level caudad, the caudad zone is the lowest instrumented vertebra and one level cephalad, and the periapical zones are the levels between the apical and cephalad/caudad zones.

\section{Statistical analysis}

Descriptive statistics were used to define the total cohort. Demographic and clinical data between the PS and the SL group were compared using the Wilcoxon rank sum test for continuous variables and the chi-square test for categorical variables. A multivariable logistical regression model was used to perform multivariable analysis. Statistical significance was defined as a $p$-value $<0.05$. SAS/JMP (SAS Institute Inc., Cary, NC, USA) was used to perform all statistical analyses.

\section{Results}

The cohort included 71 pediatric patients with AIS who had Lenke type 1 curves. The cohort included 32 Lenke 1A, 23 Lenke 1B, and 16 Lenke 1C cases. The cohort was predominantly female $(80.2 \%)$, and the average age at time of surgery was 14.2 years. Median thoracic Cobb angle for the overall cohort was $55.9^{\circ}$ (interquartile range [IQR], $50.5^{\circ}$ to $63.1^{\circ}$ ). Median bending Cobb angle was $31.9^{\circ}$ (IQR, $27.5^{\circ}$ to $38.4^{\circ}$ ). Median preoperative coronal balance was $1.15 \mathrm{~cm}$ (IQR, 0.45 to $1.87 \mathrm{~cm}$ ) between the C7 plumb line and the CSVL. Overall, the median Cobb correction for the cohort was $68.5 \%$ (IQR, $57.39 \%$ to $76.04 \%$ ), and the median coronal balance correction was $0.77 \mathrm{~cm}$ (IQR, 0.38 to $1.83 \mathrm{~cm}$ ). Median implant density for the entire construct was 1.63 (IQR, 1.36 to 1.77 ) per level fused. Median EBL for the cohort was $700 \mathrm{~mL}$ (IQR, 450 to $1,000 \mathrm{~mL}$ ) (Table 1 ).

Most of the cases, 50 (70.4\%), represented constructs utilizing only PSs in the apical and periapical zones. There were 21 (29.5\%) constructs that utilized only sublaminar wires in the apical and periapical zones. There was no significant difference in preoperative thoracic Cobb angle $\left(56.1^{\circ}\right.$ versus $\left.59.8^{\circ}, p=0.23\right)$, preoperative bending Cobb angle $\left(32.5^{\circ}\right.$ versus $\left.33.8^{\circ}, p=0.63\right)$, preoperative coronal balance ( 0.89 versus $1.25, p=0.60)$, or total implant density ( 1.58 versus $1.51, p=0.38$ ) between the SL and PS groups.

Median percent Cobb correction at one year for the PS group was $70.26 \%$ (IQR, $64.42 \%$ to $76.08 \%$ ), ranging from a median preoperative Cobb angle of $59.8^{\circ}$ to a postoperative angle of $16.5^{\circ}$. Median percent Cobb correction at one year for the SL group was 60.09 (IQR, 51.16 to 75.60), ranging from a median preoperative Cobb angle of $56.1^{\circ}$ to a postoperative angle of $22^{\circ}$. Wilcoxon rank sum test revealed a statistically significant difference in percent Cobb correction at 1 year between the SL and PS groups $(p=0.05)$ (Table 1).

Median postoperative coronal balance for the PS group and the SL group was $1.15 \mathrm{~cm}$ and $0.96 \mathrm{~cm}$, respectively. The Wilcoxon rank sum test revealed no statistically significant difference in coronal balance at 1 year between the SL and PS groups $(p=0.32)$ (Table 1). Additionally, there was no statistically significant difference in EBL between the PS and SL groups (794.8 versus $1,079, p=0.1$ ) (Table 1).

The multivariable model revealed that apical and periapical sublaminar wires were significantly negatively associated with percent Cobb correction at 1 year (coefficient $=-8.49, p=0.023$ ). Total implant density of the construct was found to be significantly positively associated with percent Cobb correction at 1 year (coefficient $=24.2$, $p<0.001)$. Preoperative bending Cobb angle, number of levels fused, and Lenke lumbar modifier were not found 
Table 1. Overview of PS and SL group demographic and clinical characteristics

\begin{tabular}{|c|c|c|c|c|}
\hline Variable & Total $(\mathrm{n}=71)$ & PS group $(n=50)$ & SL group $(n=21)$ & $p$-value \\
\hline Lenke type & & & & 0.024 \\
\hline A & 32 & 26 & 7 & \\
\hline B & 23 & 19 & 2 & \\
\hline C & 16 & 7 & 7 & \\
\hline Preoperative median thoracic Cobb angle $\left(^{\circ}\right)$ & 55.9 (IQR, 50.5 to 63.1$)$ & 59.8 & 56.1 & 0.23 \\
\hline Preoperative median bending Cobb angle $\left(^{\circ}\right)$ & 31.9 (IOR, 27.5 to 38.4$)$ & 33.8 & 32.5 & 0.63 \\
\hline Preoperative median coronal balance $(\mathrm{cm})$ & 1.15 (IQR, 0.45 to 1.87$)$ & 1.25 & 0.89 & 0.6 \\
\hline$\%$ Cobb correction & 68.5 (IQR, 57.39 to 76.04$)$ & 70.26 & 60.09 & $0.05^{*}$ \\
\hline Postoperative median thoracic Cobb angle $\left({ }^{\circ}\right)$ & 16.95 & 16.5 & 22.0 & 0.06 \\
\hline Postoperative median coronal balance $(\mathrm{cm})$ & 0.77 (IOR, 0.38 to 1.83$)$ & 1.15 & 0.96 & 0.32 \\
\hline Implant density & $1.63(\mathrm{IOR}, 1.36$ to 1.77$)$ & 1.51 & 1.58 & 0.38 \\
\hline Estimated blood loss (mL) & 700 (IOR, 450 to 1,000$)$ & 794.8 & 1,079 & 0.1 \\
\hline
\end{tabular}

PS, pedicle screw only constructs; SL, constructs using apical/periapical sublaminar wires; IQR, interquartile range. " $p<0.05$.

to be statistically significant for percent Cobb correction. In a subsequent analysis, apical/periapical sublaminar wires, total implant density, preoperative bending Cobb angle, and number of levels fused all showed no statistically significant association with postoperative coronal balance at 1 year.

\section{Discussion}

Our study revealed that, when compared to all PS constructs, the use of sublaminar wires in the apical and periapical zones of the thoracic curve was associated with reduced percent Cobb correction at 1 year. It is important to note that this relationship remains statistically significant when accounting for potential confounding factors such as total implant density, curve magnitude, curve flexibility, and lumbar modifier. Several previous studies have compared hybrid to all PS constructs, demonstrating improved ability to correct spinal deformity with PSs [1,38,14-17]. However, only two studies have compared apical sublaminar wires to apical PSs, and these studies had conflicting results $[17,18]$. It is worth noting that some thoracic curves in our cohort, specifically Lenke lumbar modifier $\mathrm{C}$ curves, may have been intentionally under corrected to preserve coronal balance. Overall coronal balance significantly improved in both of the groups, and no significant difference was found for this variable at 1 year.

In the most commonly referred to article that demon- strates the utility of apical sublaminar wires, Cheng et al. [17], reported similar percent Cobb correction between apical sublaminar wires and apical PSs. The study compared 25 patients with apical sublaminar wires and 25 patients with apical PSs. They reported percent Cobb correction of $67.4 \%$ and $68.1 \%$ in the sublaminar wire and PS groups, respectively [17]. However, the study did not include a multivariable model, and implant density was not compared between the two groups, which could have confounded the outcomes. A second study compared apical sublaminar wires, apical hooks, apical PSs, and anterior release surgery for all types of scoliosis [18]. The results of this study demonstrated improved coronal correction with PSs compared to sublaminar wires (67\% versus 52\%). This study included mostly patients with neuromuscular scoliosis, and (like the previous study) did not include multivariable analysis for overall implant density between the groups. In comparison to these studies, our results support the later of the two studies showing that apical sublaminar wires provide less coronal plan correction than PSs.

At the apex of the thoracic curve, the pedicle morphology is most altered, resulting in increased difficulty in safely placing PSs. The pedicles along the apical concavity of the deformity are the most dysplastic, and the morphology of the pedicle may not accommodate a PS. In addition to changes to the bony anatomy, the spinal cord is most at risk of injury with a medial wall breach at the concave apical pedicles $[11,12]$. These changes make the placement of 
PSs at the apex of the deformity significantly more challenging with a greater risk for complication. In situations of severe pedicle dysplasia, it may not be possible to pass a PS through the remaining pedicle, and alternative fixation is necessary [19]. Despite the decreased percent Cobb correction compared to PSs seen in this study, sublaminar wires remain an effective alternative to fixation at the apex of the thoracic curve when PS placement is not possible.

Our study is inherently limited by its sample size, specifically the number of cases utilizing wires in the apical and periapical zones, thus, forcing us to use nonparametric analyses. The study is also limited in that we do not investigate sagittal plane correction in our cohorts. Due to inability to accurately measure pre- and postoperative thoracic kyphosis from the available radiographs, this study does not investigate sagittal parameters. An additional weakness in the study is the short follow-up period of 1 year. At our institution, patients are followed up radiographically for 1 year only, and 2-year follow-up radiographs are not available. The study does not report on individual complications between the groups due to the limited reporting on complications; however, no patients in this series required a revision operation for any reason. A strength of the study is all the surgeries are performed by a single surgeon, and similar correctional maneuvers are utilized for each patient.

\section{Conclusions}

Our hypothesis was rejected, and the use of sublaminar wires at the apical and periapical zones of the spinal deformity resulted in significantly less percent Cobb correction compared to PSs. The use of either sublaminar wires or PSs resulted in a similar ability to correct coronal balance. During surgery or preoperative planning, it may be decided that PS placement is not feasible; thus, having the skill set to place sublaminar wires, sublaminar bands, and hooks remains essential. Our data support utilizing PSs at the apex and periapical vertebral levels to achieve the greatest degree of thoracic coronal correction; however, sublaminar wires are an effective and safe option to achieve appropriate coronal balance in situations where salvage fixation is required.

\section{Conflict of Interest}

No potential conflict of interest relevant to this article was reported. Valentine Esposito is supported by the National Center for Advancing Translational Sciences of the National Institutes of Health under Award number TL1TR001116. The content is solely the responsibility of the authors and does not necessarily represent the official views of the National Institutes of Health.

\section{Author Contributions}

Primary author, statistician: Valentine R. Esposito; data collection, manuscript preparation; Brian L. Dial; and principal investigator, manuscript review: Robert K. Lark.

\section{References}

1. Suk SI, Lee CK, Min HJ, Cho KH, Oh JH. Comparison of Cotrel-Dubousset pedicle screws and hooks in the treatment of idiopathic scoliosis. Int Orthop 1994;18:341-6.

2. Liljenqvist $U$, Lepsien $U$, Hackenberg L, Niemeyer T, Halm H. Comparative analysis of pedicle screw and hook instrumentation in posterior correction and fusion of idiopathic thoracic scoliosis. Eur Spine J 2002;11:336-43.

3. Kim YJ, Lenke LG, Cho SK, Bridwell KH, Sides B, Blanke K. Comparative analysis of pedicle screw versus hook instrumentation in posterior spinal fusion of adolescent idiopathic scoliosis. Spine (Phila Pa 1976) 2004;29:2040-8.

4. Dobbs MB, Lenke LG, Kim YJ, Kamath G, Peelle MW, Bridwell KH. Selective posterior thoracic fusions for adolescent idiopathic scoliosis: comparison of hooks versus pedicle screws. Spine (Phila Pa 1976) 2006;31:2400-4.

5. Hamill CL, Lenke LG, Bridwell KH, Chapman MP, Blanke K, Baldus C. The use of pedicle screw fixation to improve correction in the lumbar spine of patients with idiopathic scoliosis. Is it warranted? Spine (Phila Pa 1976) 1996;21:1241-9.

6. Di Silvestre M, Bakaloudis G, Lolli F, Vommaro F, Martikos K, Parisini P. Posterior fusion only for thoracic adolescent idiopathic scoliosis of more than 80 degrees: pedicle screws versus hybrid instrumentation. Eur Spine J 2008;17:1336-49.

7. Yilmaz G, Borkhuu B, Dhawale AA, et al. Comparative analysis of hook, hybrid, and pedicle screw instrumentation in the posterior treatment 
of adolescent idiopathic scoliosis. J Pediatr Orthop 2012;32:490-9.

8. Rafi S, Munshi N, Abbas A, Shaikh RH, Hashmi I. Comparative analysis of pedicle screw versus hybrid instrumentation in adolescent idiopathic scoliosis surgery. J Neurosci Rural Pract 2016;7:550-3.

9. Sud A, Tsirikos AI. Current concepts and controversies on adolescent idiopathic scoliosis: part II. Indian J Orthop 2013;47:219-29.

10. Crawford AH, Lykissas MG, Gao X, Eismann E, Anadio J. All-pedicle screw versus hybrid instrumentation in adolescent idiopathic scoliosis surgery: a comparative radiographical study with a minimum 2-year follow-up. Spine (Phila Pa 1976) 2013;38:1199-208.

11. Ye Q, Wu Z, Qiu G, Lin J, Wang Y, Li S. The effects of different instrumentation on scoliosis. Zhonghua Wai Ke Za Zhi 1998;36:707-10.

12. Bekki H, Harimaya K, Matsumoto Y, et al. The position of the aorta relative to the vertebrae in patients with Lenke type 1 adolescent idiopathic scoliosis. Spine (Phila Pa 1976) 2016;41:585-90.

13. Horvath MM, Winfield S, Evans S, Slopek S, Shang H, Ferranti J. The DEDUCE Guided Query tool: providing simplified access to clinical data for research and quality improvement. J Biomed Inform 2011;44:26676.
14. Bullmann V, Liljenqvist UR, Schmidt C, Schulte TL. Posterior operative correction of idiopathic scoliosis: value of pedicle screws versus hooks. Orthopade 2009;38:198-200.

15. Luo M, Li N, Shen M, Xia L. Pedicle screw versus hybrid instrumentation in adolescent idiopathic scoliosis: a systematic review and meta-analysis with emphasis on complications and reoperations. Medicine (Baltimore) 2017;96:e7337.

16. Karatoprak O, Unay K, Tezer M, Ozturk C, Aydogan $\mathrm{M}$, Mirzanli C. Comparative analysis of pedicle screw versus hybrid instrumentation in adolescent idiopathic scoliosis surgery. Int Orthop 2008;32:523-8.

17. Cheng I, Kim Y, Gupta MC, et al. Apical sublaminar wires versus pedicle screws: which provides better results for surgical correction of adolescent idiopathic scoliosis? Spine (Phila Pa 1976) 2005;30:2104-12.

18. Watanabe K, Lenke LG, Bridwell KH, et al. Comparison of radiographic outcomes for the treatment of scoliotic curves greater than 100 degrees: wires versus hooks versus screws. Spine (Phila Pa 1976) 2008;33:1084-92.

19. Sarwahi V, Sugarman EP, Wollowick AL, Amaral TD, Lo Y, Thornhill B. Prevalence, distribution, and surgical relevance of abnormal pedicles in spines with adolescent idiopathic scoliosis vs. no deformity: a CT-based study. J Bone Joint Surg Am 2014;96:e92. 